\title{
MULTIPLE SOLUTIONS OF SYSTEMS INVOLVING FRACTIONAL KIRCHHOFF-TYPE EQUATIONS WITH CRITICAL GROWTH
}

\author{
Augusto C. R. Costa and Bráulio B. V. Maia
}

Abstract. In this paper we are going to study existence and multiplicity of solutions of a system involving fractional Kirchhoff-type and critical growth of form

$$
\left\{\begin{array}{l}
M_{1}\left(\|u\|_{X}^{2}\right)(-\Delta)^{s} u=\lambda f(x, v(x))\left[\int_{\Omega} F(x, v(x)) d x\right]^{r_{1}}+|u|^{2_{s}^{*}-2} u \text { in } \Omega \\
M_{2}\left(\|v\|_{X}^{2}\right)(-\Delta)^{s} v=\gamma g(x, u(x))\left[\int_{\Omega} G(x, u(x)) d x\right]^{r_{2}}+|v|^{2_{s}^{*}-2} v \text { in } \Omega \\
u=v=0 \text { in } \mathbb{R}^{n} \backslash \Omega
\end{array}\right.
$$

where $s \in(0,1), n>2 s, \Omega \subset \mathbb{R}^{n}$ is a bounded and open set, $2_{s}^{*}=2 n /(n-2 s)$ denotes the fractional critical Sobolev exponent, the functions $M_{1}, M_{2}, f$ and $g$ are continuous functions, $(-\Delta)^{s}$ is the fractional laplacian operator, \|\|$_{X}$ is a norm in the fractional Hilbert Sobolev space $X(\Omega), F(x, v(x))=\int_{0}^{v(x)} f(\tau) d \tau, G(x, u(x))=\int_{0}^{u(x)} g(\tau) d \tau, r_{1}$ and $r_{2}$ are positive constants, $\lambda$ and $\gamma$ are real parameters. For this problem we prove the existence of infinitely many solutions, via a suitable truncation argument and exploring the genus theory introduced by Krasnoselskii. Also we show that these solutions are sufficiently regular and solve the problem pointwise.

Mathematics subject classification (2010): 35J60, 35J70, 58E05.

Keywords and phrases: Kirchhoff-type equations, fractional laplacian operator, Krasnoselskii's genus, critical growth, regularity.

\section{REFERENCES}

[1] J. G. Azorero And I. P. Alonso, Multiplicity of solutions for elliptic problems with critical exponent or with a nonsymmetric term, Trans. Amer. Math. Soc., 323, (1991), 877-895.

[2] A. Ambrosetti And P. H. Rabinowitz, Dual variational methods in critical point theory and applications, J. Functional Analysis, 14, (1973), 349-381.

[3] D. Applebaum, Lévy processes and stochastic calculus, 2nd ed., Cambridge Studies in Advanced Mathematics, Volume. 116, Cambridge Univ. Press, Cambridge, 2009.

[4] G. AUtuORI AND P. PUCCI, Elliptic problems involving the fractional Laplacian in $\mathbb{R}^{N}$, J. Differential Equations, 2558 (2013), 2340-2362.

[5] C. BAI, Multiplicity of solutions for a class of non-local elliptic operators systems, Bull. Korean Math. Soc. 54, 3 (2017), 715-729.

[6] B. Barrios, E. Colorado, R. Servadei And F. Soria, A critical fractional equation with concave-convex power nonlinearities, Ann. Inst. H. Poincaré Anal. Non Linéaire., 32 (2015) 875900.

[7] V. BENCI, On critical points theory for indefinite functionals in the presence of symmetric, Trans. Amer. Math. Soc., 274 (1982), 533-572.

[8] J. Bertoin, Lévy processes, Cambridge Tracts in Mathematics, Volume 121, 11 Cambridge Univ. Press, Cambridge, 1996.

[9] G. M. Bisci, V. D. Radulescu And R. Servadei, Variational Methods for Nonlocal Fractional Problems, Encyclopedia of Mathematics and its Applications, Cambridge University Press (2016). 
[10] G. M. BISCI AND R. SERVADEI, A bifurcation result for non-local fractional equations, Anal. Appl., 13, 4 (2015), 371-394.

[11] G. M. Bisci AND B. A. PAnsera, Three weak solutions for nonlocal fractional equations, Adv. Nonlinear Stud., 14 (2014), 591-601.

[12] G. M. BISCI AND D. REPOVS, Existence and localization of solutions for nonlocal fractional equations, Asymptot. Anal., 90 (2014), 367-378.

[13] H. BRÉZIS, Functional analysis, Sobolev spaces and partial differential equations, Universitext, Springer, New York (2011).

[14] V. I. BogacheV, Measure Theory, vol. II, Springer-Verlag, Berlin (2007).

[15] L. CAFFARELli, Non-local diffusions, drifts and games, in: Nonlinear Partial Differential Equations: The Abel Symposium 2010, Abel Symposia, H. Holden and K.H. Karlsen, eds, Volume 7, SpringerVerlag, Berlin, Heidelberg, (2012), 37-52.

[16] D. C. Clark, A variant of the Lusternik-Schnirelman theory, Indiana Univ. Math., J., 22 (1972), $65-74$.

[17] R. CONT And P. TAnKov, Financial modelling with jump processes, Chapman and Hall, CRC Financial Mathematics Series, FL, 2004.

[18] D. G. CostA, An Invitation to Variational Methods in Differential Equations, Birkhäuser,Boston (2007).

[19] A. C. R. Costa And F. R. FERreira, On a systems involving fractional Kirchhoff-type equations and Krasnoselskii's genus, Math. Methods in App. Sciences, 41, 18 (2018), 9377-9389.

[20] L. F. O. Faria, O. H. Miyagaki, F. R. Pereira, M. Squassina And C. Zhang, The BrezisNirenberg problem for nonlocal systems, Adv. Nonlinear Anal, 5 (2016), 85-103.

[21] L. F. O. FARIA, O. H. MiYAGAKI AND F. R. PEREIRA, Critical Brezis-Nirenberg problem for nonlocal systems, DOI: http://dx.doi.org/10.12775/TMNA.2017.017.

[22] A. FISCElla, Saddle point solutions for non-local elliptic operators, Topol. Methods Nonlinear Anal., 44, (2014), 527-538.

[23] A. FISCELlA, Infinitely many solutions for a critical Kirchhoff type problem involving a fractional operator, Differential Integral Equations, 5/6, 29 (2016), 513-530.

[24] A. Fiscella, G. M. Bisci AND R. SERVADEI, Bifurcation and multiplicity results for critical nonlocal fractional Laplacian problems, Bull. Sci. Math., 140, (2016), 14-35.

[25] A. FISCELla AND E. VALDINOCI, A critical Kirchhoff type problem involving a nonlocal operator, Nonlinear Anal., 94 (2014), 156-170.

[26] G. M. Figueiredo, G. M. Bisci And R. SERvadei, On a fractional Kirchhoff-type equation via Krasnoselskii's genus, Asymptot. Anal., 94 (2015), 347-361.

[27] G. M. Figueiredo And J. R. SAntos JUnior, Multiplicity of solutions for a Kirchhoff equation with sub-critical or critical growth, Differential Integral Equations 25 (2012), 853-868.

[28] M. A. KRASNOSELSKI, Topological methods in the theory of nonlinear integral equations, Mac Millan, New York (1964).

[29] O. H. MiYAGaKi AND F. R. PEREIRA, Existence results for non-local elliptic systems with nonlinearities interacting with the spectrum, Adv. in Dif. Equations, 23 (2017), 555-580.

[30] E. Di Nezza, G. Palatucci and E. Valdinoci, Hitchhiker's guide to the fractional Sobolev spaces, B.Sci. Math., 136 (2012), 521-573.

[31] G. PALATUCCI AND A. PISANTE, Improved Sobolev embeddings, profile decomposition, and concentration-compactness for fractional Sobolev spaces, Calc. Var. Partial Differential Equations, 50 (2014), 799-829.

[32] R. SERVAdei And E. VAldinoci, Mountain Pass solutions for non-local elliptic operators, J. Math. Anal. Appl., 389 (2012), 887-898. 